\title{
Variational Measurement Update for Extended Object Tracking Using Gaussian Processes
}

\author{
Murat Kumru, Hilal Köksal, Emre Özkan, Member, IEEE
}

\begin{abstract}
We present an alternative inference framework for the Gaussian process-based extended object tracking (GPEOT) models. The method provides an approximate solution to the Bayesian filtering problem in GPEOT by relying on a new measurement update, which we derive using variational Bayes techniques. The resulting algorithm effectively computes approximate posterior densities of the kinematic and the extent states. We conduct various experiments on simulated and real data and examine the performance compared with a reference method, which employs an extended Kalman filter for inference. The proposed algorithm significantly improves the accuracy of both the kinematic and the extent estimates and proves robust against model uncertainties.
\end{abstract}

Index Terms-Extended target tracking, Gaussian process, variational Bayes.

\section{INTRODUCTION}

$\mathbf{T}$ RADITIONAL object tracking is based on the pointsource model that assumes an object can generate at most one measurement per scan, [1], [2]. However, recent advances in sensor technology have led to the proliferation of applications where a single object might give rise to multiple measurements per frame. This type of problem is systematically addressed by a well-established line of research, which is referred to as extended object tracking (EOT). The objective of these methods is to estimate the object's extent, together with the kinematic variables. There have been developed many successful solutions, which rely on various formulations, e.g., [3]-[14]. A comprehensive survey of the existing literature can be found in [15] and [16].

In this paper, we focus on the Gaussian Process (GP) based EOT methods where the unknown extent is modelled by a GP prior and an approximate inference is performed to obtain its posterior. The resulting trackers estimate the kinematic state of the object while simultaneously learning its unknown extent.

The seminal model [17], here denoted as GPEOT, has been widely adopted by the research community; see, for example, [18]-[26]. However, a critical shortcoming of the current GPEOT literature is that the variety of the developed inference techniques is limited, and the resulting trackers typically rely on variants of the Kalman filter in the presence of nonlinear, implicit measurement model [23]-[26]. Although this approach has been proved to lead to a successful tracking framework, it suffers from the well-known problems of nonlinear Kalman filtering. For instance, poorly predicted state

Authors are with the Department of Electrical and Electronics Engineering, Middle East Technical University, Ankara, Turkey (e-mail: kumru@metu.edu.tr, koksal.hilal@metu.edu.tr, emreo@metu.edu.tr). This study is partially supported by Turkcell within the framework of 5G and Beyond Joint Graduate Support Programme coordinated by Information and Communication Technologies Authority. density and high levels of uncertainty in the model might radically degrade the estimation performance. These limitations potentially impede the widespread use of the method in various tracking applications.

In this letter, we propose an inference method for the GPEOT model. The suggested method is based on an analytical measurement update, which is derived using the variational Bayes techniques. The resulting algorithm constitutes a recursive tracking filter, which is shown to significantly improve tracking performance and be robust against model uncertainties via comprehensive experiments.

\section{Proposed Method}

GPEOT aims at estimating unknown contours of dynamic objects. The key idea behind the GPEOT approach is to describe the extent of a star-convex ${ }^{1}$ object by a radial function $f(\theta)$ which is assumed to have a GP prior, i.e., $f(\theta) \sim \mathcal{G} \mathcal{P}\left(0, k\left(\theta, \theta^{\prime}\right)\right)$, where $k\left(\theta, \theta^{\prime}\right)$ is the covariance function, which basically computes the covariance between the function evaluations at angles $\theta$ and $\theta^{\prime}$, [17], [27]. To facilitate an efficient, recursive inference mechanism, the GP model is approximated for the function values $\mathbf{f} \triangleq\left[f\left(\theta_{1}^{\mathbf{f}}\right) \ldots f\left(\theta_{M}^{\mathbf{f}}\right)\right]^{\top}$ at some basis inputs. The resulting measurement model, which relates the latent variables to a point measurement $\mathbf{z}_{k, l}$, is given in the following.

$$
\begin{aligned}
\mathbf{z}_{k, l} & =\mathbf{c}_{k}+\tilde{H}_{l}^{\mathbf{f}}\left(\mathbf{c}_{k}, \psi_{k}\right) \mathbf{f}_{k}+\mathbf{e}_{k, l} \\
& =\mathbf{h}_{k, l}\left(\mathbf{x}_{k}\right)+\mathbf{e}_{k, l}, \quad \mathbf{e}_{k, l} \sim \mathcal{N}\left(0, R_{k, l}\right),
\end{aligned}
$$

where $\mathbf{c}_{k}$ is a reference point (referred to as object center) within the body of the object; $\psi_{k}$ denotes the object orientation, $\mathbf{x}_{k}$ is a unified state vector including both the kinematic and the extent variables, and $\mathbf{e}_{k, l} \sim \mathcal{N}\left(0, R_{k, l}\right)$ denotes the zero-mean Gaussian measurement noise with covariance $R_{k, l}$. Further details on the variables used in (1a) can be found in the supplementary material. The representation for the complete measurement set $\left\{\mathbf{z}_{k, l}\right\}_{l=1}^{n_{k}}$ collected at time $k$ is formed as

$$
\begin{aligned}
\mathbf{z}_{k} & =\left[\mathbf{z}_{k, 1}^{\top}, \ldots, \mathbf{z}_{k, n_{k}}^{\top}\right]^{\top}, R_{k}=\operatorname{diag}\left[R_{k, 1}, \ldots, R_{k, n_{k}}\right], \\
\mathbf{h}_{k}\left(\mathbf{x}_{k}\right) & =\left[\mathbf{h}_{k, 1}\left(\mathbf{x}_{k}\right)^{\top}, \ldots, \mathbf{h}_{k, n_{k}}\left(\mathbf{x}_{k}\right)^{\top}\right]^{\top},
\end{aligned}
$$

where diag[.] returns a block diagonal matrix $R_{k}$ by placing the matrices $\left\{R_{k, l}\right\}_{l=1}^{n_{k}}$ along the diagonal.

It is possible to construct a state space model by combining the given measurement equation with a dynamic model of the state vector, and treat the estimation problem in the Bayesian framework.

${ }^{1}$ A set $\mathcal{S}$ is called star-convex if each line segment from the origin to any point in $\mathcal{S}$ is fully contained in $\mathcal{S}$. 


\section{A. Variational Measurement Update}

Suppose at time $k$, we have the following predicted density for the kinematic and the extent states

$p\left(\overline{\mathbf{x}}_{k}, \mathbf{f}_{k} \mid \mathbf{z}_{1: k-1}\right)=\mathcal{N}\left(\overline{\mathbf{x}}_{k} ; \boldsymbol{\mu}_{k \mid k-1}^{x}, P_{k \mid k-1}^{x}\right) \mathcal{N}\left(\mathbf{f}_{k} ; \boldsymbol{\mu}_{k \mid k-1}^{f}, P_{k \mid k-1}^{f}\right)$,

where $\boldsymbol{\mu}_{k \mid k-1}$ and $P_{k \mid k-1}$ denote the predicted mean and covariance, respectively. $\overline{\mathbf{x}}_{k}$ indicates the kinematic state, which includes the object center $\mathbf{c}_{k}$, the orientation $\psi_{k}$, and the linear and angular velocities $\mathbf{x}_{k}^{*}$, i.e., $\overline{\mathbf{x}}_{k}=\left[\mathbf{c}_{k}^{\top} \psi_{k}\left(\mathbf{x}_{k}^{*}\right)^{\top}\right]^{\top}$, and $\mathbf{f}_{k}$ is the extent state. Considering the model in (1), the measurement likelihood function can be written as

$$
p\left(\mathbf{z}_{k} \mid \overline{\mathbf{x}}_{k}, \mathbf{f}_{k}\right)=\mathcal{N}\left(\mathbf{z}_{k} ; \mathbf{h}_{k}\left(\mathbf{x}_{k}\right), R_{k}\right),
$$

where $\mathbf{x}_{k}$ is the unified state vector including the kinematic and the extent state, i.e., $\mathbf{x}_{k}=\left[\begin{array}{ll}\overline{\mathbf{x}}_{k}^{\top} & \mathbf{f}_{k}^{\top}\end{array}\right]^{\top}$. In Bayesian filtering, the aim is to obtain the posterior density $p\left(\overline{\mathbf{x}}_{k}, \mathbf{f}_{k} \mid \mathbf{z}_{1: k}\right)$. Following the classical variational Bayes approach, we approximate the posterior as $p\left(\overline{\mathbf{x}}_{k}, \mathbf{f}_{k} \mid \mathbf{z}_{1: k}\right) \approx q\left(\overline{\mathbf{x}}_{k}, \mathbf{f}_{k}\right)=$ $q_{x}\left(\overline{\mathbf{x}}_{k}\right) q_{f}\left(\mathbf{f}_{k}\right)$, where $q_{x}\left(\overline{\mathbf{x}}_{k}\right)$ and $q_{f}\left(\mathbf{f}_{k}\right)$ denote the factorized densities for the kinematic and the extent state, respectively.

The variational formulation seeks for the approximate posterior that minimize the Kullback-Leibler (KL) divergence between the true and the approximate posterior, and the solution satisfies the following equations, [28, Ch. 10],

$$
\begin{aligned}
\log q_{x}\left(\overline{\mathbf{x}}_{k}\right) & =\mathbb{E}_{q_{f}}\left[\log p\left(\mathbf{z}_{k}, \overline{\mathbf{x}}_{k}, \mathbf{f}_{k} \mid \mathbf{z}_{1: k-1}\right)\right]+c_{x}, \\
\log q_{f}\left(\mathbf{f}_{k}\right) & =\mathbb{E}_{q_{x}}\left[\log p\left(\mathbf{z}_{k}, \overline{\mathbf{x}}_{k}, \mathbf{f}_{k} \mid \mathbf{z}_{1: k-1}\right)\right]+c_{f},
\end{aligned}
$$

where $c_{x}$ and $c_{f}$ stand for the collection of terms that are not functions of the corresponding factor. The estimated densities are obtained by iteratively computing one of the factors while keeping the other fixed at its most recent estimate, [28, Ch. 10]. These fixed-point iterations are guaranteed to converge to a solution that corresponds to a local minimum of the KL divergence, [29, Sec. 2.5].

To obtain analytical expressions for (4), we first need to express the joint density $p\left(\mathbf{z}_{k}, \overline{\mathbf{x}}_{k}, \mathbf{f}_{k} \mid \mathbf{z}_{1: k-1}\right)$ as

$$
p\left(\mathbf{z}_{k}, \overline{\mathbf{x}}_{k}, \mathbf{f}_{k} \mid \mathbf{z}_{1: k-1}\right)=p\left(\mathbf{z}_{k} \mid \overline{\mathbf{x}}_{k}, \mathbf{f}_{k}\right) p\left(\overline{\mathbf{x}}_{k}, \mathbf{f}_{k} \mid \mathbf{z}_{1: k-1}\right) .
$$

Next, we will compute the estimated densities at the $(i+1)^{t h}$ iteration, which are denoted by $q_{x}^{(i+1)}\left(\overline{\mathbf{x}}_{k}\right)$ and $q_{f}^{(i+1)}\left(\mathbf{f}_{k}\right)$.

1) Computation of $q_{x}^{(i+1)}\left(\overline{\boldsymbol{x}}_{k}\right)$ : Substituting (5) in (4a), we can write

$$
\begin{aligned}
\log q_{x}^{(i+1)}\left(\overline{\mathbf{x}}_{k}\right)=\mathbb{E}_{q_{f}^{(i)}} & {\left[\log p\left(\mathbf{z}_{k} \mid \overline{\mathbf{x}}_{k}, \mathbf{f}_{k}\right)\right] } \\
& +\mathbb{E}_{q_{f}^{(i)}}\left[\log p\left(\overline{\mathbf{x}}_{k} \mid \mathbf{z}_{1: k-1}\right)\right]+c_{x} .
\end{aligned}
$$

Unfortunately, this expression does not yield a Gaussian density for $q_{x}^{(i+1)}$ due to the nonlinearity in the mean of $p\left(\mathbf{z}_{k} \mid \overline{\mathbf{x}}_{k}, \mathbf{f}_{k}\right)$. To alleviate this problem, we approximate the measurement model by its first-order Taylor series expansion.

$$
\begin{aligned}
\mathbf{z}_{k} & =\mathbf{h}_{k}\left(\boldsymbol{\mu}_{x}^{(i)}, \boldsymbol{\mu}_{f}^{(i)}\right)+A^{(i)}\left(\overline{\mathbf{x}}_{k}-\boldsymbol{\mu}_{x}^{(i)}\right)+B^{(i)}\left(\mathbf{f}_{k}-\boldsymbol{\mu}_{f}^{(i)}\right)+\mathbf{e}_{k} \\
& =\mathbf{g}^{(i)}+A^{(i)} \overline{\mathbf{x}}_{k}+B^{(i)} \mathbf{f}_{k}+\mathbf{e}_{k} \\
\mathbf{g}^{(i)} & \triangleq \mathbf{h}_{k}\left(\boldsymbol{\mu}_{x}^{(i)}, \boldsymbol{\mu}_{f}^{(i)}\right)-A^{(i)} \boldsymbol{\mu}_{x}^{(i)}-B^{(i)} \boldsymbol{\mu}_{f}^{(i)} \\
A^{(i)} & \left.\triangleq \frac{\partial \mathbf{h}_{k}}{\partial \overline{\mathbf{x}}_{k}}\right|_{\overline{\mathbf{x}}_{k}=\boldsymbol{\mu}_{x}^{(i)}, \mathbf{f}_{k}=\boldsymbol{\mu}_{f}^{(i)},\left.B^{(i)} \triangleq \frac{\partial \mathbf{h}_{k}}{\partial \mathbf{f}_{k}}\right|_{\overline{\mathbf{x}}_{k}=\boldsymbol{\mu}_{x}^{(i)}, \mathbf{f}_{k}=\boldsymbol{\mu}_{f}^{(i)}}}
\end{aligned}
$$

Note that the Taylor series approximation is performed within every VB iteration which can break the convergence results of the VB. However, we did not observe any divergence tendency of the algorithm in our experiments with simulated and real data.

Consequently, we can write $\mathbb{E}_{q_{f}^{(i)}}\left[\log p\left(\mathbf{z}_{k} \mid \overline{\mathbf{x}}_{k}, \mathbf{f}_{k}\right)\right]$ as

$$
\begin{aligned}
& \mathbb{E}_{q_{f}^{(i)}}\left[\log p\left(\mathbf{z}_{k} \mid \overline{\mathbf{x}}_{k}, \mathbf{f}_{k}\right)\right] \\
& =-0.5\left(\mathbf{z}_{k}-\mathbf{g}^{(i)}-A^{(i)} \overline{\mathbf{x}}_{k}\right)^{\top} R_{k}^{-1}\left(\mathbf{z}_{k}-\mathbf{g}^{(i)}-A^{(i)} \overline{\mathbf{x}}_{k}\right) \\
& \left.\quad-2\left(\mathbf{z}_{k}-\mathbf{g}^{(i)}-A^{(i)} \overline{\mathbf{x}}_{k}\right)^{\top} R_{k}^{-1} B \boldsymbol{\mu}_{f}^{(i)}\right)+c_{x} .
\end{aligned}
$$

It can be shown that the variational iterate in (6) becomes a Gaussian distribution, i.e., $q_{x}^{(i+1)}\left(\overline{\mathbf{x}}_{k}\right)=\mathcal{N}\left(\boldsymbol{\mu}_{x}^{(i+1)}, P_{x}^{(i+1)}\right)$, and its mean and covariance can be computed by a Kalman filter measurement update as follows.

$$
\begin{aligned}
& \boldsymbol{\mu}_{x}^{(i+1)}=\boldsymbol{\mu}_{k \mid k-1}^{x}+K_{k}\left(\mathbf{z}_{k}-\mathbf{g}^{(i)}-B^{(i)} \boldsymbol{\mu}_{f}^{(i)}-A^{(i)} \boldsymbol{\mu}_{k \mid k-1}^{x}\right) \\
& P_{x}^{(i+1)}=P_{k \mid k-1}^{x}-K_{k} A^{(i)} P_{k \mid k-1}^{x} \\
& S_{k}=A^{(i)} P_{k \mid k-1}^{x}\left(A^{(i)}\right)^{\top}+R_{k}, K_{k}=P_{k \mid k-1}^{x}\left(A^{(i)}\right)^{\top}\left(S_{k}\right)^{-1}
\end{aligned}
$$

2) Computation of $q_{f}^{(i+1)}\left(\boldsymbol{f}_{k}\right)$ : From (4b) and (5), it follows

$$
\begin{aligned}
\log q_{f}^{(i+1)}\left(\mathbf{f}_{k}\right)= & \mathbb{E}_{q_{x}^{(i+1)}}\left[\log p\left(\mathbf{z}_{k} \mid \overline{\mathbf{x}}_{k}, \mathbf{f}_{k}\right)\right] \\
& +\mathbb{E}_{q_{x}^{(i+1)}}\left[\log p\left(\mathbf{f}_{k} \mid \mathbf{z}_{1: k-1}\right)\right]+c_{f} .
\end{aligned}
$$

By using the measurement model in (1), we can write

$$
\begin{aligned}
& \mathbb{E}_{q_{x}^{(i+1)}}\left[\log p\left(\mathbf{z}_{k} \mid \overline{\mathbf{x}}_{k}, \mathbf{f}_{k}\right)\right]=-0.5(\mathbf{f}_{k}^{\top} \underbrace{\stackrel{\underbrace{\triangleq g_{1}\left(\overline{\mathbf{x}}_{k}\right)}_{\left[\tilde{H}\left(\overline{\mathbf{x}}_{k}\right)^{\top} R_{k}^{-1} \tilde{H}\left(\overline{\mathbf{x}}_{k}\right)\right.}}{\mathbf{f}_{k}}}_{\triangleq \Delta} \mathbf{f}_{k} \\
& -2 \mathbf{f}_{k}^{\top} \underbrace{\triangleq \overbrace{\tilde{H}\left(\overline{\mathbf{x}}_{k}\right)^{\top} R_{k}^{-1}\left(\mathbf{z}_{k}-\mathbf{c}_{k}\right)}}_{\triangleq \delta}])+c_{f}
\end{aligned}
$$

The expectations indicated by $\Delta$ and $\delta$ include highly nonlinear functions of $\overline{\mathbf{x}}_{k}$, and do not end up with compact analytical expressions. We employ the unscented transformation [30] to approximately compute these expectations as

$$
\Delta \approx \sum_{j=0}^{M} \pi^{j} g_{1}\left(\overline{\mathbf{x}}_{k}^{j}\right), \quad \boldsymbol{\delta} \approx \sum_{j=0}^{M} \pi^{j} g_{2}\left(\overline{\mathbf{x}}_{k}^{j}\right),
$$

where $\left\{\overline{\mathbf{x}}_{k}^{j}\right\}_{j=0}^{M}$ and $\left\{\pi^{j}\right\}_{j=0}^{M}$ denote the sigma-points and the associated weights, which are explicitly defined in the supplementary material due to page limitations.

The resulting expression in (11) together with the prediction density imply that the variational iterate for the extent is a Gaussian density, and we can analytically compute its mean $\boldsymbol{\mu}_{f}^{(i+1)}$ and covariance $P_{f}^{(i+1)}$ as follows

$$
\begin{aligned}
& \boldsymbol{\mu}_{f}^{(i+1)}=\left(\Delta+\left(P_{k \mid k-1}^{f}\right)^{-1}\right)^{-1}\left(\boldsymbol{\delta}+\left(P_{k \mid k-1}^{f}\right)^{-1} \boldsymbol{\mu}_{k \mid k-1}^{f}\right), \\
& P_{f}^{(i+1)}=\left(\Delta+\left(P_{k \mid k-1}^{f}\right)^{-1}\right)^{-1} .
\end{aligned}
$$

This concludes the derivation of the variational approximation-based measurement update. This mechanism, 


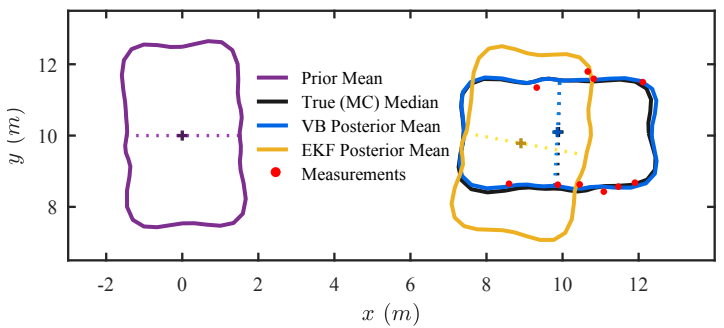

Fig. 1. Illustration of a single measurement update. We also obtain the median of the true posterior distribution by using 1 million Monte Carlo (MC) samples, which is plotted in black. The number of variational iterations is 10 for VB. (The plus signs and the dashed lines indicate the estimates of the object center and the orientation, respectively.)

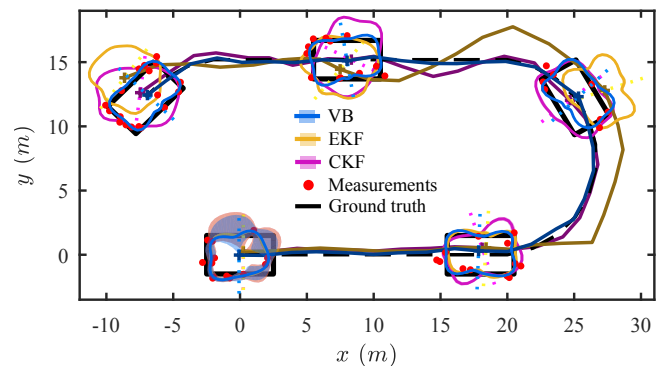

Fig. 2. A typical realization of S2-Exp2 for a rectangular object.

combined with a process model of the state vector, establishes an EOT approach; and we will examine the performance of the corresponding algorithm in the following sections.

\section{B. A Closer Look to a Single Measurement Update}

Before proceeding to a detailed performance analysis, we first want to demonstrate the capabilities of the proposed method, denoted as 'VB', and the reference method [17], named 'EKF'. For this purpose, we focus on a single measurement update that is realized for the setting in Fig. 1.

In this example, the underlying object has a rectangular extent. Both methods are initialized by the same prior and are provided with the same set of measurements. We compare the resulting posterior distributions. Contrary to EKF, the VB algorithm performs multiple iterations in a single measurement update. Consequently, the posterior obtained by VB is significantly closer to the true posterior compared to that of EKF. This desirable behavior is the primary motivation behind the development of $\mathrm{VB}$, and it will be demonstrated to lead to superior tracking performance in Section III.

\section{EXPERIMENTAL RESUlts}

\section{A. Experiments with Simulated Data}

We conduct various experiments with simulated data considering different motion behaviors and extent models. Measurements are randomly generated from the underlying object contour and corrupted by Gaussian noise with covariance $R=0.3^{2} I_{2} \mathrm{~m}^{2}$. The number of the measurements on each scan is sampled from a Poisson distribution with mean 15.

To assess the performance of the algorithms, we regard the Intersection-Over-Union (IOU) measure as in [17]. As a complementary performance measure, we also compute the root mean squared error (RMSE) of the object orientation.

Time evolution of the kinematics is expressed by the nearly constant velocity model. We describe the dynamics of the extent state by the following model, which provides the maximum entropy prediction density for unknown and slowly varying dynamics, [19, Theorem 1].

$$
\mathbf{f}_{k+1}=\mathbf{f}_{k}+\mathbf{w}_{k}^{f}, \mathbf{w}_{k}^{f} \sim \mathcal{N}\left(\mathbf{0}, Q^{f}\right), Q^{f}=\left(\lambda^{-1}-1\right) P_{k \mid k}^{f},
$$

where $P_{k \mid k}^{f}$ denotes the covariance of the estimated extent, and $\lambda<1$ is a factor that scales up the estimated covariance to compute the predicted covariance, i.e, $P_{k+1 \mid k}^{f}=\lambda^{-1} P_{k \mid k}^{f}$. Both of the methods employ the same GP model defined by the periodic covariance function, [17]. The number of the variational iterations in VB is set to 10 for all experiments. Additionally, we implement another reference method, denoted as 'CKF', based on the cubature Kalman filter [31].

We investigate two distinct scenarios, which are indicated by S1 and S2. In S1 (model match scenario), the motion of the object is simulated by the nearly constant velocity model, and the trackers are provided with the true parameters of motion. In S2, the object traverses the path that is depicted in Fig. 2 at a constant speed $v$. In this setting, we examine a model mismatch between the true and the assumed motion dynamics.

For both of the scenarios, we construct three separate experiments, denoted Exp1, Exp2 and Exp3, which present varying degrees of difficulty for a tracking application. More specifically, Exp1 corresponds to the least challenging environment by using a sufficiently small sampling time and involving modest levels of uncertainty in object's motion. In Exp2, the object of interest has greater maneuverability (e.g., using larger process noise for kinematics in S1, and increased speed in S2). Exp3 has the same motion characteristics with Exp1; however, the measurements are acquired less frequently as the sampling time is increased. Finally, for each experiment, we use three different extent models, i.e., rectangular (T1), triangular (T2) and a random extent sampled from a GP prior (T3). Due to page limitations, we present the full details of the simulation parameters in the supplementary material.

TABLE I

Mean Value of the INTERSECTION-OVER-Union (IOU)

\begin{tabular}{|c|c|c|c|c|c|c|c|}
\hline & \multicolumn{3}{|c|}{ S1 } & \multicolumn{3}{|c|}{ S2 } \\
\hline & & T1 & T2 & T3 & T1 & T2 & T3 \\
\hline \multirow{3}{*}{$\overline{\mathbf{x}}$} & VB & 0.880 & 0.866 & 0.940 & 0.884 & 0.866 & 0.916 \\
\hline & EKF & 0.875 & 0.863 & 0.937 & 0.882 & 0.869 & 0.913 \\
\hline & CKF & 0.834 & 0.851 & 0.936 & 0.867 & 0.870 & 0.921 \\
\hline \multirow{3}{*}{ 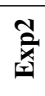 } & VB & 0.838 & 0.777 & 0.929 & 0.829 & 0.784 & 0.881 \\
\hline & EKF & 0.569 & 0.557 & 0.827 & 0.691 & 0.650 & 0.805 \\
\hline & CKF & 0.637 & 0.596 & 0.778 & 0.677 & 0.676 & 0.803 \\
\hline \multirow{3}{*}{ 藏 } & VB & 0.814 & 0.783 & 0.918 & 0.827 & 0.766 & 0.869 \\
\hline & EKF & 0.655 & 0.631 & 0.830 & 0.696 & 0.644 & 0.795 \\
\hline & CKF & 0.667 & 0.597 & 0.710 & 0.613 & 0.491 & 0.633 \\
\hline
\end{tabular}

TABLE II

RMSE OF THE ORIENTATION ANGLE [RAD]

\begin{tabular}{|c|c|c|c|c|c|c|c|}
\hline & \multicolumn{3}{|c|}{ S1 } & \multicolumn{3}{|c|}{ S2 } \\
\hline & & T1 & T2 & T3 & T1 & T2 & T3 \\
\hline \multirow{3}{*}{ 忞 } & VB & 0.084 & 0.051 & 0.046 & 0.076 & 0.046 & 0.057 \\
\hline & EKF & 0.094 & 0.052 & 0.046 & 0.082 & 0.044 & 0.060 \\
\hline & CKF & 0.310 & 0.087 & 0.050 & 0.092 & 0.045 & 0.063 \\
\hline \multirow{3}{*}{ 彭 } & VB & 0.314 & 0.443 & 0.090 & 0.183 & 0.224 & 0.110 \\
\hline & EKF & 1.200 & 1.060 & 0.399 & 0.850 & 0.534 & 0.389 \\
\hline & CKF & 1.382 & 1.197 & 0.840 & 0.783 & 0.433 & 0.393 \\
\hline \multirow{3}{*}{ 商 } & VB & 0.409 & $\mathbf{0 . 3 8 7}$ & $\mathbf{0 . 0 8 2}$ & 0.209 & $\mathbf{0 . 3 2 5}$ & 0.212 \\
\hline & EKF & 0.997 & 0.844 & 0.438 & 0.913 & 0.569 & 0.401 \\
\hline & CKF & 1.373 & 1.349 & 1.257 & 1.549 & 1.542 & 1.358 \\
\hline
\end{tabular}



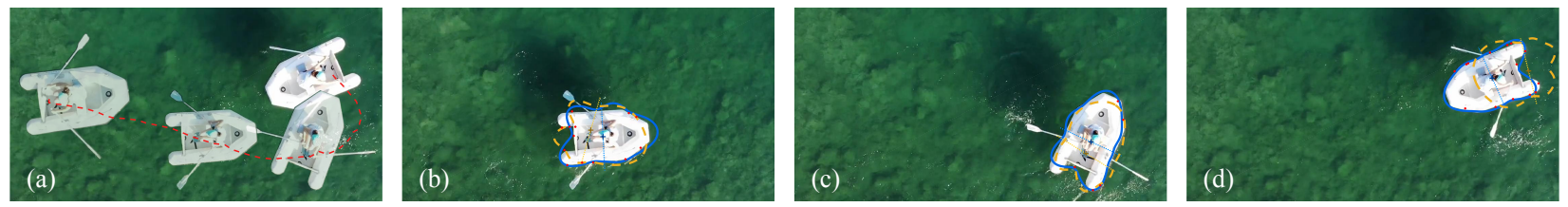

Fig. 3. Tracking results for the video experiment. Fig. (a) shows the outline of the experiment. Starting from the leftmost position, the dinghy moves along the red dashed line. In Figs. (b)-(d), the estimates of the algorithms are plotted for three different frames (VB: - EKF: = ", measurements: •).

The mean of the IOU values and the RMSE of the object orientation are given in Tables I and II, respectively. All numbers are averaged over 100 Monte Carlo runs with randomly generated measurements (for all experiments), trajectories (S1), and object extents (T3).

The results suggest that all methods are equally successful in Exp1. However, in Exp2 and Exp3, VB is significantly superior in regard to the estimation performance for the extent and the orientation. This observation is valid for both scenarios, S1 and S2, concerning the settings with model match and model mismatch. An example realization of the u-turn scenario with higher speed (S2-Exp2) using the rectangular extent model is illustrated in Fig. 2. Accordingly, we observe that VB establishes a more robust tracking algorithm at the cost of more computations. This can be attributed to the iterative nature of $\mathrm{VB}$, which benefits multiple linearizations and expectation computations in a single measurement update.

\section{B. Experiments with Real Data}

In this section, the performance of the algorithms is demonstrated using real data. In particular, we investigate two experiments regarding different sensor modalities. Table III presents the corresponding results.

Experiment with Camera Data: In the first experiment, we consider information collected by a camera, mounted on an airborne vehicle which is steady over a surveillance region. The experiment takes place in a cove located at the western Mediterranean coast of Turkey. The scene involves a dinghy exhibiting agile maneuvers as shown in Fig. 3(a). Throughout the scenario, the camera captures an image in every 2 seconds. There are various algorithms that can extract features from camera images, such as Harris corner detector [32] and Lowe's Scale Invariant Feature Transform (SIFT) [33] (for an overview, see [34, Ch. 4]). To investigate a more general case, we consider point measurements generated by uniformly sampling the object's contour. The results obtained by using the features extracted by the Harris corner detector closely follow the results presented here, but they are not included in the manuscript because of the page limitations. The number of measurements on each frame is sampled from a Poisson distribution with average 10. Both methods share the identical set of parameters, which are specified in the supplementary material.

Figs. 3(b)-(d) visualize the outputs of the algorithms for three instants. At the beginning of the experiment, while the dinghy moves along a relatively straight line, both methods perform successfully. However, once the vehicle starts maneuvering, VB exhibits favorable performance in terms of both the extent and the kinematic estimates as seen in Figs. (c) and (d). (a)

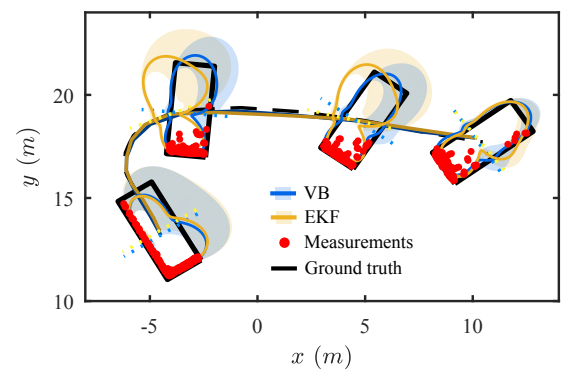

(b)
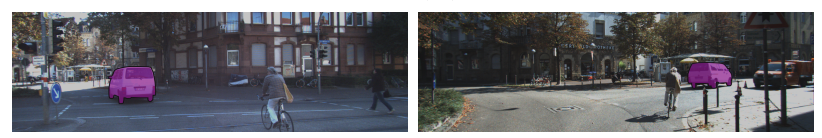

Fig. 4. Fig. (a): Tracking results for the Kitti experiment (shaded areas represent 1-std confidence interval of the corresponding estimate). Fig. (b): Example video frames from the experiment.

TABLE III

RESULTS FOR THE EXPERIMENTS WITH REAL DATA

\begin{tabular}{|l|c|c|c|c|}
\cline { 2 - 5 } \multicolumn{1}{c|}{} & \multicolumn{2}{c|}{ Video Data } & \multicolumn{2}{c|}{ Laser Scanner Data } \\
\cline { 2 - 5 } \multicolumn{1}{c|}{} & IOU & RMSE $(\hat{\psi})[\mathbf{r a d}]$ & IOU & RMSE $(\hat{\psi})[\mathbf{r a d}]$ \\
\hline VB & $\mathbf{0 . 8 0 4}$ & $\mathbf{0 . 1 0 5}$ & $\mathbf{0 . 7 6 2}$ & $\mathbf{0 . 0 4 6}$ \\
\hline EKF & 0.672 & 0.363 & 0.624 & 0.276 \\
\hline
\end{tabular}

Experiment with Laser Scanner Data: In the second experiment, we extract a sequence of point measurements acquired by a Velodyne HDL-64E laser scanner included in Kitti tracking benchmark, [35]. The outline of the scenario is illustrated by two example video frames in Fig. 4(b). The experiment takes place on an urban street where the ego and the target vehicles exhibit turn maneuvers while moving in the same direction. Both methods use the same set of parameters, which are given in the supplementary material.

The results are demonstrated in Fig. 4(a). Both VB and EKF satisfactorily estimate the trajectory. Throughout the scenario, the sensor is mainly able to observe the left and rear parts of the target, thus the algorithms appropriately decrease the uncertainty on the observed portion of the extent while a larger uncertainty is associated with the unobserved section. However, during the maneuvering phase, VB outperforms EKF in orientation estimation.

\section{CONCLUSION}

We derived an alternative inference method for the GPEOT models using the variational Bayes techniques. The resulting algorithm is shown to significantly improve the tracking performance through experiments on simulated and real data (e.g., the percentage improvements in the IOU measure and in the accuracy of the orientation estimates exceed $40 \%$ and $400 \%$, respectively). Additionally, it proves to be more robust for the scenarios with moderate or high levels of uncertainty included in the model. In this regard, we believe that the suggested method will enable the utilization of the GPEOT trackers in a wider range of real-world applications. 


\section{REFERENCES}

[1] Y. Bar-Shalom, X. R. Li, and T. Kirubarajan, Estimation with Applications to Tracking and Navigation: Theory, Algorithms and Software. John Wiley \& Sons, 2004.

[2] S. R. Jondhale and R. S. Deshpande, "Kalman filtering framework-based real time target tracking in wireless sensor networks using generalized regression neural networks," IEEE Sensors J., vol. 19, pp. 224-233, Jan. 2019.

[3] J. W. Koch, "Bayesian approach to extended object and cluster tracking using random matrices," IEEE Trans. Aerosp. Electron. Syst., vol. 44, pp. 1042-1059, Jul. 2008.

[4] M. Feldmann, D. Franken, and W. Koch, "Tracking of extended objects and group targets using random matrices," IEEE Trans. Signal Process., vol. 59, pp. 1409-1420, Apr. 2011.

[5] M. Baum and U. D. Hanebeck, "Extended object tracking with random hypersurface models," IEEE Trans. Aerosp. Electron. Syst., vol. 50, pp. 149-159, Jan. 2014.

[6] C. Lundquist, U. Orguner, and F. Gustafsson, "Extended target tracking using polynomials with applications to road-map estimation," IEEE Trans. Signal Process., vol. 59, pp. 15-26, Jan. 2010.

[7] J. Lan and X. R. Li, "Tracking of extended object or target group using random matrix -Part II: Irregular object," in Proc. Int. Conf. Inf. Fusion (FUSION), (Singapore), Jul. 2012.

[8] K. Granström, C. Lundquist, and U. Orguner, "Tracking rectangular and elliptical extended targets using laser measurements," in Proc. Int. Conf. Inf. Fusion (FUSION), (Chicago, IL, USA), Jul. 2011.

[9] U. Orguner, "A variational measurement update for extended target tracking with random matrices," IEEE Trans. Signal Process., vol. 60, pp. 3827-3834, Jul. 2012.

[10] A. Zea, F. Faion, M. Baum, and U. D. Hanebeck, "Level-set random hypersurface models for tracking nonconvex extended objects," IEEE Trans. Aerosp. Electron. Syst., vol. 52, pp. 2990-3007, Dec. 2016.

[11] W. Cao, J. Lan, and X. R. Li, "Extended object tracking and classification using radar and ESM sensor data," IEEE Signal Process. Lett., vol. 25, pp. 90-94, Jan. 2018.

[12] H. Kaulbersch, J. Honer, and M. Baum, "A cartesian B-spline vehicle model for extended object tracking," in Proc. Int. Conf. Inf. Fusion (FUSION), (Cambridge, UK), Jul. 2018.

[13] S. Kraemer, C. Stiller, and M. E. Bouzouraa, "LiDAR-based object tracking and shape estimation using polylines and free-space information," in Proc. IEEE/RSJ Int. Conf. Intell. Robots and Syst. (IROS), (Madrid, Spain), Oct. 2018.

[14] J. Lan and X. R. Li, "Extended-object or group-target tracking using random matrix with nonlinear measurements," IEEE Trans. Signal Process., vol. 67, pp. 5130-5142, Oct. 2019.

[15] K. Granström, M. Baum, and S. Reuter, "Extended object tracking: Introduction, overview and applications," J. Adv. Inf. Fusion, vol. 12, pp. 139-174, Dec. 2017.

[16] L. Mihaylova, A. Y. Carmi, F. Septier, A. Gning, S. K. Pang, and S. Godsill, "Overview of Bayesian sequential Monte Carlo methods for group and extended object tracking," Digital Signal Process., vol. 25, pp. 1-16, Feb. 2014.
[17] N. Wahlström and E. Özkan, "Extended target tracking using Gaussian processes," IEEE Trans. Signal Process., vol. 63, pp. 4165-4178, Aug. 2015.

[18] T. Hirscher, A. Scheel, S. Reuter, and K. Dietmayer, "Multiple extended object tracking using Gaussian processes," in Proc. Int. Conf. Inf. Fusion (FUSION), (Heidelberg, Germany), Jul. 2016.

[19] E. Özkan, N. Wahlström, and S. J. Godsill, "Rao-Blackwellised particle filter for star-convex extended target tracking models," in Proc. Int. Conf. Inf. Fusion (FUSION), (Heidelberg, Germany), Jul. 2016.

[20] B. Tuncer, M. Kumru, and E. Özkan, "Extended target tracking and classification using neural networks," in Proc. Int. Conf. Inf. Fusion (FUSION), (Ottawa, ON, Canada), Jul. 2019.

[21] Y. Guo, Y. Li, A. Xue, R. Tharmarasa, and T. Kirubarajan, "Simultaneous tracking of a maneuvering ship and its wake using Gaussian processes," Signal Process., vol. 172, Jul. 2020.

[22] M. Michaelis, P. Berthold, D. Meissner, and H.-J. Wuensche, "Heterogeneous multi-sensor fusion for extended objects in automotive scenarios using Gaussian processes and a GMPHD-filter," in Proc. IEEE ISIF Workshop Sens. Data Fusion: Trends, Solutions, Appl. (SDF), (Bonn, Germany), Oct. 2017.

[23] S. Lee and J. McBride, "Extended object tracking via positive and negative information fusion," IEEE Trans. Signal Process., vol. 67, pp. 1812-1823, Feb. 2019.

[24] K. Thormann, M. Baum, and J. Honer, "Extended target tracking using Gaussian processes with high-resolution automotive radar," in Proc. Int. Conf. Inf. Fusion (FUSION), (Cambridge, UK), Jul. 2018.

[25] M. Kumru and E. Özkan, "3D extended object tracking using recursive Gaussian processes," in Proc. Int. Conf. Inf. Fusion (FUSION), (Cambridge, UK), Jul. 2018.

[26] B. Tuncer, M. Kumru, E. Özkan, and A. A. Alatan, "Extended object tracking and shape classification," in Proc. Int. Conf. Inf. Fusion (FUSION), (Cambridge, UK), Jul. 2018.

[27] C. E. Rasmussen and C. K. Williams, Gaussian Processes for Machine Learning. Cambridge, MA, USA: MIT Press, 2006.

[28] C. M. Bishop, Pattern Recognition and Machine Learning. Springer, 2006.

[29] D. M. Blei, A. Kucukelbir, and J. D. McAuliffe, "Variational inference: A review for statisticians," J. Amer. Statistical Assoc., vol. 112, no. 518, pp. 859-877, 2017.

[30] S. J. Julier and J. K. Uhlmann, "Unscented filtering and nonlinear estimation," Proceedings of the IEEE, vol. 92, pp. 401-422, Mar. 2004.

[31] I. Arasaratnam and S. Haykin, "Cubature Kalman filters," IEEE Trans. Autom. Cont., vol. 54, no. 6, pp. 1254-1269, 2009.

[32] C. G. Harris, M. Stephens, et al., "A combined corner and edge detector," in Proc. Alvey Vis. Conf., 1988.

[33] D. G. Lowe, "Distinctive image features from scale-invariant keypoints," Int. J. Comput. Vis., vol. 60, no. 2, pp. 91-110, 2004.

[34] R. Szeliski, Computer Vision: Algorithms and Applications. Springer, 2010.

[35] A. Geiger, P. Lenz, and R. Urtasun, "Are we ready for autonomous driving? The KITTI vision benchmark suite," in Proc. IEEE Conf. Comput. Vis. Pattern Recogn. (CVPR), (Providence, RI, USA), Jun. 2012. 


\section{Variational Measurement Update for Extended Object Tracking Using Gaussian Processes}

\author{
Supplementary Material
}

In this supplementary material, we provide the details of the measurement model and the employed unscented transform, and we reveal additional details of the experiments considered in the article. The references included in this document are as in the article.

\section{A. Details of the Measurement Model}

The variables used in the measurement model (1a) are explicitly defined as follows, [17].

$$
\tilde{H}_{l}^{\mathbf{f}}\left(\mathbf{c}_{k}, \psi_{k}\right)=\mathbf{p}_{k, l} H_{k, l}^{\mathbf{f}}, R_{k, l}=\mathbf{p}_{k, l} R_{k, l}^{\mathbf{f}} \mathbf{p}_{k, l}^{\top}+R
$$

where $H_{k, l}^{\mathbf{f}}=k\left(\theta_{k, l}, \boldsymbol{\theta}^{\mathbf{f}}\right) K\left(\boldsymbol{\theta}^{\mathbf{f}}, \boldsymbol{\theta}^{\mathbf{f}}\right)^{-1}, R_{k, l}^{\mathbf{f}}=k\left(\theta_{k, l}, \theta_{k, l}\right)-$ $H_{k, l}^{\mathbf{f}} k\left(\boldsymbol{\theta}^{\mathbf{f}}, \theta_{k, l}\right), \mathbf{p}_{k, l}=\left[\cos \left(\theta_{k, l}^{\mathrm{G}}\right) \sin \left(\theta_{k, l}^{\mathrm{G}}\right)\right]^{\top}, \theta_{k, l}=\theta_{k, l}^{\mathrm{G}}-$ $\psi_{k}, \theta_{k, l}^{\mathrm{G}}=\angle\left(\mathbf{z}_{k, l}-\mathbf{c}_{k}\right), \boldsymbol{\theta}^{\mathbf{f}} \triangleq\left[\theta_{1}^{\mathbf{f}} \ldots \theta_{M}^{\mathbf{f}}\right]^{\top}$ includes the basis input angles, and the $i$ - $j^{\text {th }}$ entry of the covariance matrix is $K\left(\boldsymbol{\theta}^{\mathbf{f}}, \boldsymbol{\theta}^{\mathbf{f}}\right)_{i j}=k\left(\theta_{i}^{\mathbf{f}}, \theta_{j}^{\mathbf{f}}\right)$.

\section{B. The Unscented Transformation}

In Section II-A2 of the paper, the following intractable expectations are approximately computed by the unscented transformation as

$$
\begin{gathered}
\mathbb{E}_{q_{x}^{(i+1)}}[\underbrace{\tilde{H}\left(\overline{\mathbf{x}}_{k}\right)^{\top} R_{k}^{-1} \tilde{H}\left(\overline{\mathbf{x}}_{k}\right)}_{g_{1}\left(\overline{\mathbf{x}}_{k}\right)}] \approx \sum_{j=0}^{M} \pi^{j} g_{1}\left(\overline{\mathbf{x}}_{k}^{j}\right) \\
\mathbb{E}_{q_{x}^{(i+1)}}[\underbrace{\tilde{H}\left(\overline{\mathbf{x}}_{k}\right)^{\top} R_{k}^{-1}\left(\mathbf{z}_{k}-\mathbf{c}_{k}\right)}_{g_{2}\left(\overline{\mathbf{x}}_{k}\right)}] \approx \sum_{j=0}^{M} \pi^{j} g_{2}\left(\overline{\mathbf{x}}_{k}^{j}\right),
\end{gathered}
$$

where $\left\{\overline{\mathbf{x}}_{k}^{j}\right\}_{j=0}^{M}$ and $\left\{\pi^{j}\right\}_{j=0}^{M}$ denote the sigma-points and the associated weights, and $q_{x}^{(i+1)}\left(\overline{\mathbf{x}}_{k}\right)$ is the variational iterate for the kinematic state defined as $q_{x}^{(i+1)}\left(\overline{\mathbf{x}}_{k}\right)=\mathcal{N}\left(\boldsymbol{\mu}_{x}^{(i+1)}, P_{x}^{(i+1)}\right)$.

In the literature, there are various methods to select the sigma-points and their weights. In this study, we follow the standard approach in [30] and specify

$\overline{\mathbf{x}}_{k}^{j \triangleq}\left\{\begin{array}{ll}\boldsymbol{\mu}_{x}^{(i+1)}, & j=1 \\ \boldsymbol{\mu}_{x}^{(i+1)}+\left[\sqrt{\frac{m}{1-\bar{\pi}} P_{x}^{(i+1)}}\right]_{:, j-1}, & 2 \leq j \leq m+1 \\ \boldsymbol{\mu}_{x}^{(i+1)}-\left[\sqrt{\frac{m}{1-\bar{\pi}} P_{x}^{(i+1)}}\right]_{:, j-m-1}, & m+2 \leq j \leq 2 m+1\end{array}\right.$, $\pi^{j} \triangleq\left\{\begin{array}{ll}\bar{\pi}, & j=1 \\ \frac{1-\bar{\pi}}{2 m}, & 2 \leq j \leq 2 m+1\end{array}\right.$,

where $m$ is dimension of the kinematic state vector, $[\cdot]_{:, j}$ denotes the $j^{\text {th }}$ column of the matrix argument, $\sqrt{ } \cdot$ indicates the matrix square root operation, and we choose $\bar{\pi}=\frac{1}{2 m+1}$.

\section{Additional Details of the Experiments}

To assess the performance of the proposed method, we regard the Intersection-over-Union (IOU) and the root mean squared error (RMSE) of the object orientation. The definitions of these measures are given in the following: $\operatorname{IOU}(\hat{S}, S)=$
TABLE SI

Motion VARIABles USED IN THE EXPERIMENTS WITH Simulated DATA

\begin{tabular}{|c|c|c|c|c|c|c|c|}
\cline { 2 - 8 } \multicolumn{1}{c|}{} & \multicolumn{3}{c|}{ S1 } & \multicolumn{4}{c|}{ S2 } \\
\cline { 2 - 8 } \multicolumn{1}{c|}{} & $T(\mathrm{~s})$ & $\sigma_{c}(\mathrm{~m})$ & $\sigma_{\psi}(\mathrm{rad})$ & $v(\mathrm{~m} / \mathrm{s})$ & $T(\mathrm{~s})$ & $\sigma_{c}(\mathrm{~m})$ & $\sigma_{\psi}(\mathrm{rad})$ \\
\hline Exp1 & 1 & 0.1 & 0.01 & 0.5 & 1 & 0.1 & 0.01 \\
\hline Exp2 & 1 & 1 & 0.1 & 2 & 1 & 0.5 & 0.05 \\
\hline Exp3 & 4 & 0.1 & 0.01 & 0.5 & 4 & 0.1 & 0.01 \\
\hline
\end{tabular}

$\operatorname{area}(\hat{S} \cap S) / \operatorname{area}(\hat{S} \cup S), \operatorname{RMSE}(\hat{\psi})=\sqrt{\frac{1}{N} \sum_{k=1}^{N}\left(\hat{\psi}_{k}-\psi_{k}\right)^{2}}$, where the estimated and the true extent are represented by $\hat{S}$ and $S$, respectively; $\hat{\psi}$ and $\psi$ indicate the estimated and the true orientation angle, respectively.

As specified in the paper, the nearly constant velocity model is used to describe the time evolution of the kinematic state $\overline{\mathbf{x}}_{k}$. The description of the model is as follows.

$$
\begin{aligned}
\overline{\mathbf{x}}_{k+1} & =\bar{F} \overline{\mathbf{x}}_{k}+\overline{\mathbf{w}}_{k}, \quad \overline{\mathbf{w}}_{k} \sim \mathcal{N}(\mathbf{0}, \bar{Q}), \\
\bar{F} & =\left[\begin{array}{cc}
1 & T \\
0 & 1
\end{array}\right] \otimes I_{3}, \quad \bar{Q}=\left[\begin{array}{cc}
\frac{T^{3}}{3} & \frac{T^{2}}{2} \\
\frac{T^{2}}{2} & T
\end{array}\right] \otimes\left[\begin{array}{ccc}
\sigma_{c}^{2} & 0 & 0 \\
0 & \sigma_{c}^{2} & 0 \\
0 & 0 & \sigma_{\psi}^{2}
\end{array}\right],
\end{aligned}
$$

where $T$ is the sampling time; $\sigma_{c}$ and $\sigma_{\psi}$ indicate the process noise standard deviations for the center and the orientation, respectively; $\otimes$ denotes the Kronecker product.

The definition of the periodic covariance function of the Gaussian process (GP) is given by $k\left(\theta, \theta^{\prime}\right)=$ $\sigma_{f}^{2} \exp \left(-\frac{2 \sin ^{2}\left(\frac{\left(\theta-\theta^{\prime}\right)}{2}\right)}{l^{2}}\right)+\sigma_{r}^{2}$; where $\sigma_{f}^{2}$ is the prior variance, $l$ is the length-scale, and $\sigma_{r}^{2}$ is the constant term.

\section{Values of the Variables Used in the Experiments}

1) Experiments with Simulated Data: Table SI declares the values of the motion variables used in the experiments with simulated data. In particular, the given numbers specify both the simulation generation process and the parameters used by the trackers. As the first scenario (S1) examines a model match setting, the values of the motion variables, which are used while generating the ground truth, are identically provided to the trackers.

The hyperparameters of the GP model are set to $\sigma_{f}=2 \mathrm{~m}$, $\sigma_{r}=0.6 \mathrm{~m}$ and $l=\pi / 6 \mathrm{rad}$; and the number of basis points is 50 , which are evenly spaced in $[0,2 \pi] . \lambda=0.99$ is used for the extent dynamics.

2) Experiments with Real Data:

- Experiment with Camera Data

The values of the parameters used in the experiment with camera data are as follows. The sampling time is set to $T=2 \mathrm{~s} ; R=3^{2} I_{2} \mathrm{~m}^{2}$ is the measurement noise covariance matrix; the process noise standard deviations are used as $\sigma_{c}=1 \mathrm{~m}, \sigma_{\psi}=0.2 \mathrm{rad}$; the same hyperparameters as defined in the simulation experiments are utilized for the GP model except $\sigma_{f}=20 \mathrm{~m}$.

- Experiment with Laser Scanner Data

The values of the parameters used in the experiment with camera data are as follows. The sampling time is set to $T=0.5 \mathrm{~s} ; R=0.8^{2} I_{2} \mathrm{~m}^{2}$ is the measurement noise covariance matrix; the process noise standard deviations are used as $\sigma_{c}=3 \mathrm{~m}, \sigma_{\psi}=0.8 \mathrm{rad}$; the same hyperparameters as defined in the simulation experiments are utilized for the GP model except $l=\pi / 5 \mathrm{rad}$. 